\title{
Cold neutral gas associated with the Gum nebula
}

\author{
E.M. Reynoso ${ }^{\star}$ and G.M. Dubner ${ }^{\star \star}$ \\ Instituto de Astronomía y Física del Espacio, C.C.67, Suc. 28, 1428 Buenos Aires, Argentina.
}

Received May 28; accepted September 6, 1996

\begin{abstract}
Based on HI maps covering the 3600 square degrees area delimited by $230^{\circ} \leq l \leq 290^{\circ},-30^{\circ} \leq b \leq$ $30^{\circ}$, we investigated the neutral hydrogen distribution in the Gum nebula and its environments. The main result is the appearence of a huge disk of neutral gas at a kinematical distance compatible with that of the nebula, and whose angular dimensions coincide with the optical image of the Gum nebula. The disk, roughly centered at $l \simeq 262^{\circ}, b \simeq-3^{\circ}$, is located at $\sim 500 \mathrm{pc}$ and has a radius of $\sim 150 \mathrm{pc}$. We propose that this HI disk is the neutral gas component of the optical nebula, hence becoming the first time in which a structure with the dimensions of the optical nebula is detected in other wavelengths. We analyze the fitting of observations with different theoretical models and conclude that the most plausible interpretation is that the Gum nebula is a supershell produced by the action of repeated SN explosions, where the explosions may have originated in the aged association Vela OB2. In this context, the thick HI shell shown by Dubner et al. (1992) near $\left(266^{\circ},-2.5^{\circ}\right)$ and the Vela SNR are the debris of two of the several outbursts which started in the region about $610^{6}$ years ago. The southern boundary is distorted due to the presence of $\mathrm{a} \sim 28^{\circ}$ radius shell, which appears to be an interstellar bubble driven by the stellar winds of the O-type star HD 49798.
\end{abstract}

Key words: ISM: supernova remnants - ISM: individual objects: Gum nebula — ISM: bubbles — radio lines: ISM

\section{Introduction}

The Gum nebula is the largest $\mathrm{H} \alpha$ emission object in angular dimensions detected in our Galaxy, and extends over most of the Vela-Puppis region in the southern sky. From $\mathrm{H} \alpha$ images, it is described as a $36^{\circ}$ diameter sphere centered at $l=258^{\circ}, b=-2^{\circ}$ (Chanot \& Sivan 1983).

Send offprint requests to: E.M. Reynoso

* Fellowship of CONICET, Argentina.

** Member of the Carrera del Investigador Científico of CONICET, Argentina
Distance estimates range from 300 pc (Franco 1990) to 800 pc (Sahu 1992).

The nature of the Gum nebula is still a controversial matter. The models proposed to explain its origin include a fossil Strömgren sphere (Brandt et al. 1971), a classical H II region (Gum 1956; Beuerman 1973), a supernova remnant (Reynolds 1976a,b), a wind driven bubble (Weaver et al. 1977), a supershell produced by a combination of stellar winds and supernovæ explosions (McCray 1988) and a "blister" in the near edge of a giant molecular cloud caused by the Vela R2 association (Sahu 1992).

There have been some attempts to determine whether or not the gas associated to the Gum nebula is in expansion. Based on $\mathrm{H} \alpha$, [N II], [O IV] and He I emission lines, Reynolds (1976a) concludes that the Gum nebula is expanding with a velocity of $20 \mathrm{~km} \mathrm{~s}^{-1}$. However, Wallerstein et al. (1980) and Hippelein \& Weinberg (1975) could not arrive to the same conclusion based on Ca II and Na I optical data. Srinivasan et al. (1987) found an expansion velocity of $\leq 10 \mathrm{~km} \mathrm{~s}^{-1}$ based on [N II] and $\mathrm{H} \alpha$ data.

The first insight into the distribution of neutral gas in the Gum nebula has been performed by McGee et al. (1963, 1966). They found a ridge of enhanced emission at zero velocity following closely the $\mathrm{H} \alpha$ circular envelope. Dubner et al. (1992) mapped the area delimited by $250^{\circ} \leq l \leq 274^{\circ},-10^{\circ} \leq b \leq+5^{\circ}$ combining observations performed with the $30 \mathrm{~m}$ IAR radiotelescope, and data from the HI $21 \mathrm{~cm}$ line survey of Strong et al. (1982), taken with the $64 \mathrm{~m}$ Parkes radiotelescope. Based on these images, they show the existence of a $7^{\circ}$ radius thick shell at a distance compatible with that of the Gum nebula. The shell, centered at $\left(266^{\circ},-2.5^{\circ}\right)$ and expanding at $v \simeq 8 \mathrm{~km} \mathrm{~s}^{-1}$, appears poorly correlated with the optical emission.

In this paper, the study is extended to the area delimited by $230^{\circ} \leq l \leq 290^{\circ},-30^{\circ} \leq b \leq+30^{\circ}$ so as to investigate the characteristics of the neutral gas in the whole nebula and its surroundings in search of a possible neutral gas counterpart for the optical nebula. For this purpose, we combined the surveys performed by Weaver \& Williams (1973), Heiles \& Habing (1974), Colomb et al. 
$(1977,1980)$ and Strong et al. (1982) in the HI $21 \mathrm{~cm}$ line. The procedure to obtain the maps was not straightforward because of the irregular sampling of the sky for latitudes $|b| \geq 10^{\circ}$. In Sect. 2, the observational data bases and the procedure followed for the construction of the maps are described. Based on the present data, possible explanations for the origin of the Gum nebula are investigated.

\section{Observations and maps}

In order to cover the 3600 square degrees area contained between $230^{\circ} \leq l \leq 290^{\circ},-30^{\circ} \leq b \leq+30^{\circ}$, four different data sets were used. The strip delimited by $b=-10^{\circ}$ and $+10^{\circ}$ was sampled by the surveys of Weaver \& Williams (1973) from $l=230^{\circ}$ to $250^{\circ}$, and Strong et al. (1982) from $245^{\circ}$ to $290^{\circ}$. The region outside this band was surveyed by Heiles \& Habing (1974) and Colomb et al. (1977, 1980). In Fig. 1, the observed points in each survey are plotted. Regions of overlap among the different surveys were used to control the quality of the match. Since several regions appear quite under-sampled, unpredictable errors can be produced after the processing and combination of the data. Therefore, we avoid the analysis of small scale structure. In what follows, a brief description of the different surveys is given:

a) Weaver \& Williams (1973): this atlas covers a regular grid in $l, b$, with an interval of $0.5^{\circ}$ in longitude and $0.25^{\circ}$ in latitude. Observations were performed over an area of $10^{\circ} \leq l \leq 250^{\circ},-10^{\circ} \leq b \leq+10^{\circ}$, using the $26 \mathrm{~m}$ telescope of Hat Creek, Berkeley, with an angular resolution of $35.5^{\prime}$. The noise level was $0.38 \mathrm{~K}$ in antenna temperature. The relation between antenna and brightness temperature is $T_{\mathrm{B}} / T_{\mathrm{A}}=(1.18 \pm 0.085)$. The profiles consist of 200 velocity channels spaced in $\Delta v=1.055 \mathrm{~km} \mathrm{~s}^{-1}$ (except for the extreme channels, not used in the present study, for which $\Delta v$ is different), and the reference velocity, which in general varies for each profile, is referred to channel 122 .

b) Heiles \& Habing (1974): observations were also made with the $26 \mathrm{~m}$ telescope of Hat Creek, Berkeley, in the region observable from the local latitude and for $|b|>10^{\circ}$. In this case, the sampling was irregular in $l, b$, with $\Delta l=0.3^{\circ} / \cos b$ and $\Delta b=0.6^{\circ}$. The southern limit of the sample was $\delta=-30^{\circ}$. The noise level was in general less than $0.4 \mathrm{~K}$. The data are given in antenna temperature, with $T_{\mathrm{B}} / T_{\mathrm{A}} \simeq 1.145$. The profiles, with 100 velocity channels, have a velocity interval of $\Delta v=1.05 \mathrm{~km} \mathrm{~s}^{-1}$, except for the first eight and last five channels, for which $\Delta v=6.3 \mathrm{~km} \mathrm{~s}^{-1}$. The reference velocity, $0 \mathrm{~km} \mathrm{~s}^{-1}$ in almost all the profiles, is refered to channel 52 .

c) Colomb et al. $(1977,1980)$ : observations were taken with the $30 \mathrm{~m}$ radiotelescope of the IAR, with a resolution of $34^{\prime}$ at $1420 \mathrm{MHz}$. This survey has been performed with the purpose of being combined with the survey of Heiles and Habing, giving a complete coverage of the sky at $|b|>10^{\circ}$. The data were obtained by scanning in Right
Ascention for fixed declination, with $\Delta \delta=1^{\circ}$ between scans. The velocity resolution is $2 \mathrm{~km} \mathrm{~s}^{-1}$. The velocity range is about $80 \mathrm{~km} \mathrm{~s}^{-1}$, and the first channel velocity varies from one profile to the other. The data are given in antenna temperature, with $T_{\mathrm{B}} / T_{\mathrm{A}} \simeq 1.145$.

d) Strong et al. (1982): observations were taken with the $64 \mathrm{~m}$ radiotelescope at Parkes, with an angular resolution of $15^{\prime}$ at $1420 \mathrm{MHz}$. The spectral resolution was $0.824 \mathrm{~km} \mathrm{~s}^{-1}$, and the number of velocity channels, 512. This survey covers the area $245^{\circ} \leq l \leq 12^{\circ}$, $-10^{\circ} \leq b \leq+10^{\circ}$, with a regular sampling in galactic coordinates with $\Delta l=0.5^{\circ}$ and $\Delta b=1^{\circ}$. The reference velocity, at the central channel, is $0 \mathrm{~km} \mathrm{~s}^{-1}$, except for the strip delimited by $245^{\circ} \leq l \leq 326^{\circ},-2^{\circ} \leq b \leq+2^{\circ}$, where this velocity is $+50 \mathrm{~km} \mathrm{~s}^{-1}$. The noise level is $1 \mathrm{~K}$. Due to the rather low signal-to-noise ratio, a Hanning smoothing was applied, which lowered the velocity resolution of the profiles to $1.648 \mathrm{~km} \mathrm{~s}^{-1}$ and the rms noise level to $0.6 \mathrm{~K}$. The data were corrected for a systematic shift of $2.6 \mathrm{~km} \mathrm{~s}^{-1}$ in velocity (Dubner et al. 1992). No beam smoothing was applied.

All data were converted into brightness temperature and fitted into one single field, within the velocity range $-44 \leq v \leq+46 \mathrm{~km} \mathrm{~s}^{-1}$, in $2 \mathrm{~km} \mathrm{~s}^{-1}$ velocity intervals, with a uniform grid in galactic coordinates. Each grid point profile was calculated as the weighted mean among the four nearest profiles (one per quadrant). In the zone of overlap between the Strong et al. and the Weaver \& Williams surveys, only profiles from the former were used. The final product was a set of spectral data placed each $0.5^{\circ}$ in $l$ and $1^{\circ}$ in $b$. The images were processed using the AIPS software. The rms noise level of each channel is better than $1 \mathrm{~K}$.

\section{Results}

After inspection of the images produced, we found no traces of neutral gas which could be related to the Gum nebula except for the lowest LSR velocity channels. In Fig. 2 we show $l, b$ maps between -2 and $+20 \mathrm{~km} \mathrm{~s}^{-1}$, which include both greyscale and line contours. One of the most conspicuous features is a bright, extended circular structure, $6.5^{\circ}$ in radius, centered approximately at $l \simeq 266^{\circ}, b \simeq-2.5^{\circ}$, and showing a hole in the HI distribution at the center. This structure, appearing between -2 and $+12 \mathrm{~km} \mathrm{~s}^{-1}$, has already been discussed in Dubner et al. (1992), under the denomination of "thick HI shell". The distance to the thick HI shell was estimated to be $(500 \pm 300) \mathrm{pc}$, its expansion velocity in 6 to $8 \mathrm{~km} \mathrm{~s}^{-1}$, and its mass in more than $2.110^{4} M_{\odot}$. It has been proposed that the thick HI shell is the remnant of an old SN explosion. It has also been suggested that this shell may be related to the Gum nebula. We will come back to this point in Sect. 4.

At $v=+6 \mathrm{~km} \mathrm{~s}^{-1}$, a large disk $\sim 34^{\circ}$ in diameter, roughly centered at $l=262^{\circ}, b=-3^{\circ}$, can be observed. 


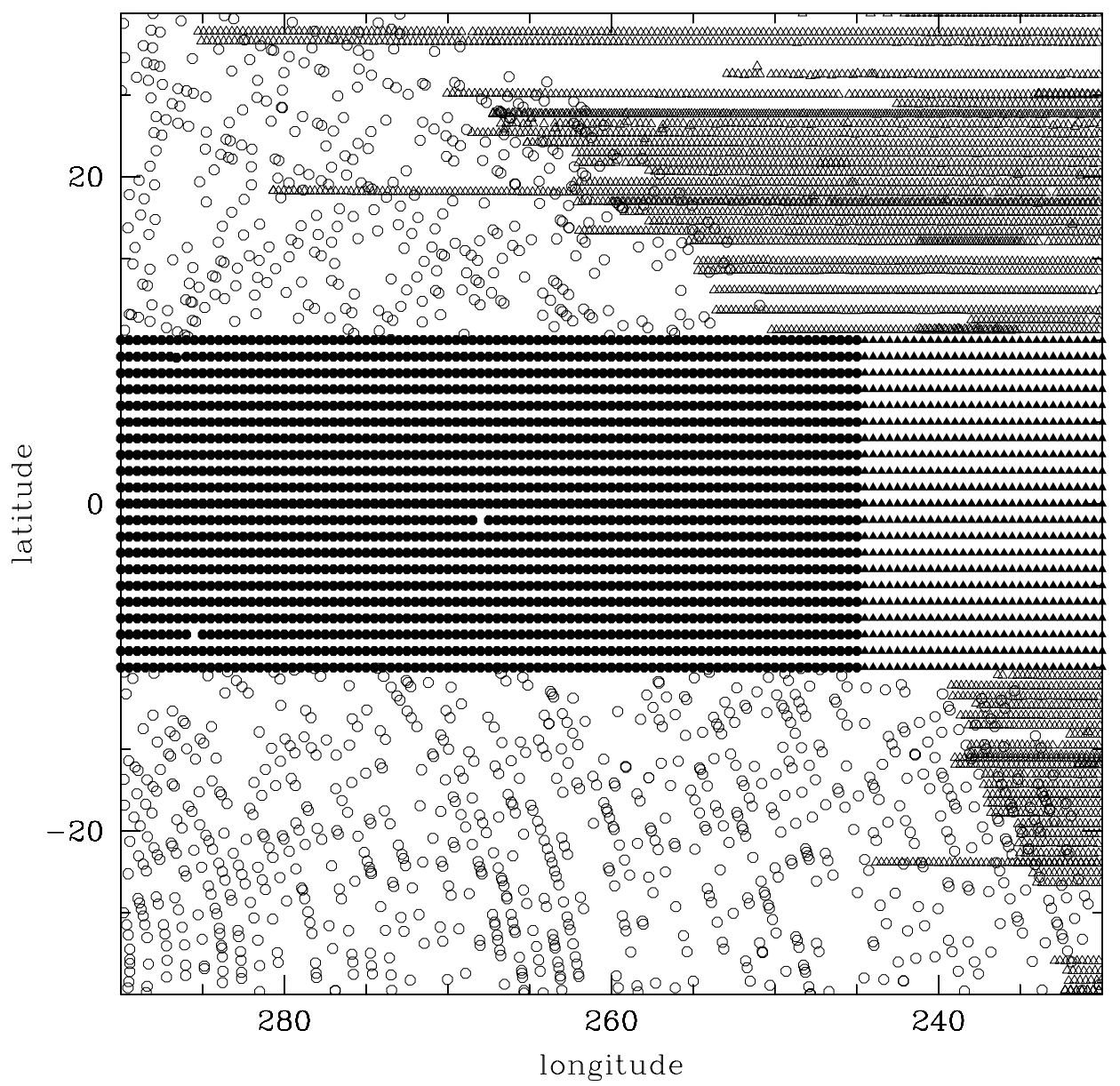

Fig. 1. Distribution of the observed points in each survey. Open triangles correspond to the survey of Heiles \& Habing (1974); open circles, to Colomb et al. $(1977,1980)$; filled circles, to Strong et al. (1982); and filled triangles, to Weaver \& Williams (1973)

This structure is also seen at +8 and $+10 \mathrm{~km} \mathrm{~s}^{-1}$; at $v=+12 \mathrm{~km} \mathrm{~s}^{-1}$ it has almost disappeared. This large disk will be called hereafter the "giant HI disk", and will be shown to agree in position and size with the optical image of the Gum nebula. The southern hemisphere of the giant $\mathrm{HI}$ disk is not well defined due to the presence of another shell, $\sim 28^{\circ}$ in diameter, centered at $l=257^{\circ}, b=-23^{\circ}$, which appears from -10 to $+14 \mathrm{~km} \mathrm{~s}^{-1}$.

From $v=+12$ to $v=+16 \mathrm{~km} \mathrm{~s}^{-1}$, a tail at $l=260^{\circ}$ can be seen emerging northwards from the giant HI disk. At the same position, Chanot \& Sivan (1983) have found faint, diffuse $\mathrm{H} \alpha$ wisps extending from the main body of the Gum nebula.

The giant HI disk is probably also present at LSR velocities lower than $6 \mathrm{~km} \mathrm{~s}^{-1}$, but confusion with local gas makes its recognition more difficult. It seems reasonable to consider this structure as extending from 0 to $+10 \mathrm{~km} \mathrm{~s}^{-1}$. If we assume that the giant HI disk is undergoing a symmetric expansion, we can roughly estimate its systemic velocity as $+5 \mathrm{~km} \mathrm{~s}^{-1}$ with an expansion velocity of about $5 \mathrm{~km} \mathrm{~s}^{-1}$. In Fig. 3, we show an integrated map between
0 and $+10 \mathrm{~km} \mathrm{~s}^{-1}$, in which the giant $\mathrm{HI}$ disk and the thick HI shell can be easily distinguished. To the left of the disk (longitudes higher than $270^{\circ}$ ), the emission at the galactic plane is brighter than to the right (longitudes lower than $240^{\circ}$ ) due to the effects of velocity crowding in the fourth galactic quadrant near $270^{\circ}$. Figure 4 shows an overlap of the $\mathrm{H} \alpha$ optical image of the Gum nebula with some contours of the HI emission. The $\mathrm{H} \alpha$ digitalized image used here was produced from the photograph taken by Sivan (1974). The white stars indicate the positions of $\gamma^{2}$ Velorum $\left(l=262.8^{\circ}, b=-7.69^{\circ}\right)$ and $\zeta$ Puppis $\left(l=255.58^{\circ}, b=-4.42^{\circ}\right)$.

We have estimated the mass of the giant HI disk in $\sim 5.510^{5} d^{2} M_{\odot}$, where $d$ is the distance in kpc. According to the galactic rotation model of Fich et al. (1989), the kinematical distance corresponding to the center of the giant HI disk at a velocity of $+5 \mathrm{~km} \mathrm{~s}^{-1}$ is $\sim 580 \mathrm{pc}$, with an uncertainty of about $20 \%$. It should be kept in mind that kinematical distances are strongly sensitive to the galactic longitude in this part of the galaxy. Therefore, we can consider that, within the errors, both the giant 

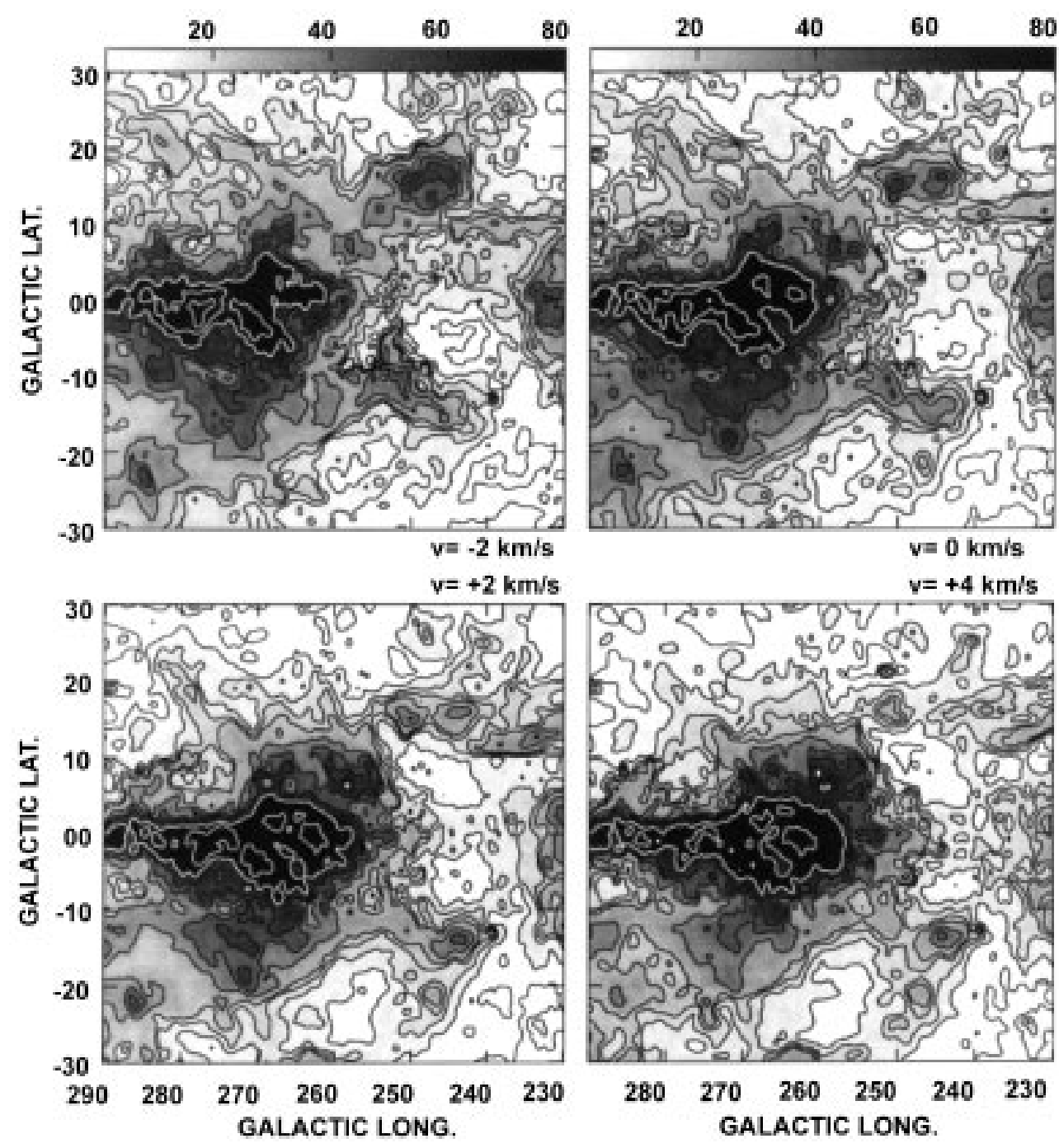

Fig. 2. Greyscale $l, b$ maps of the $21 \mathrm{~cm}$ emission between -2 and $20 \mathrm{~km} \mathrm{~s}^{-1}$, given in brightness temperature. The black contours, uniform in the whole velocity range, are at 4, 8, 12, $1620,30,40$ and $50 \mathrm{~K}$. The white contours are at 70,100 and $130 \mathrm{~K}$. The greyscale levels are shown on top of the first two images. The LSR velocity is indicated

HI disk and the thick HI shell share a distance of about $(500 \pm 100) \mathrm{pc}$. In this case, the linear radius corresponding to the giant HI disk turns out to be $\sim 150 \mathrm{pc}$. The mass of the giant $\mathrm{HI}$ disk can be estimated to be $\sim 1.410^{5} M_{\odot}$, and the expansion kinetic energy, in $\sim 3.410^{49}$ ergs.

\section{Discussion}

\subsection{Comparison with emission at other wavelengths}

A comparison between the $\mathrm{H} \alpha$ image of the Gum nebula as taken from Sivan (1974) with the giant HI disk (Fig. 1) shows a good positional coincidence between the neutral gas disk and the optical emission, as well as a similar angular size. To the northwest, the HI emission slightly departs from the circular symmetry. The two optical wings between $b \simeq+10^{\circ}$ and $b \simeq+15^{\circ}$ lie outside the edge of the disk. The eastern wing depicts a good morphological correspondence with the HI contours. The other wing, however, appears detached from the giant HI disk.

The Puppis-Vela region between $240^{\circ} \leq l \leq 275^{\circ}$, $-20^{\circ} \leq b \leq+20^{\circ}$ was thoroughly studied at $12,25,60$ and $100 \mu \mathrm{m}$ by Sahu (1992) using IRAS data. At 25, 60 and $100 \mu \mathrm{m}$, the author finds a $7.5^{\circ}$ radius shell centered at $l=263^{\circ}, b=-7^{\circ}$, which is called the "IRAS Vela Shell". The center of this structure is coincident with $\gamma^{2}$ Velorum and with the Vela OB2 association. Thus, it is assumed that the IRAS Vela Shell has the same distance as the Vela OB2 assocation, i.e. 450 pc. We checked the $100 \mu \mathrm{m}$ image presented by Sahu (1992) and found a remarkably good positional correspondence between the inner thick HI shell reported by Dubner et al. (1992) and the infrared emission. In both cases, the emission encloses a minimum at $265^{\circ},-5^{\circ}$. Therefore, the northern part of the IRAS 

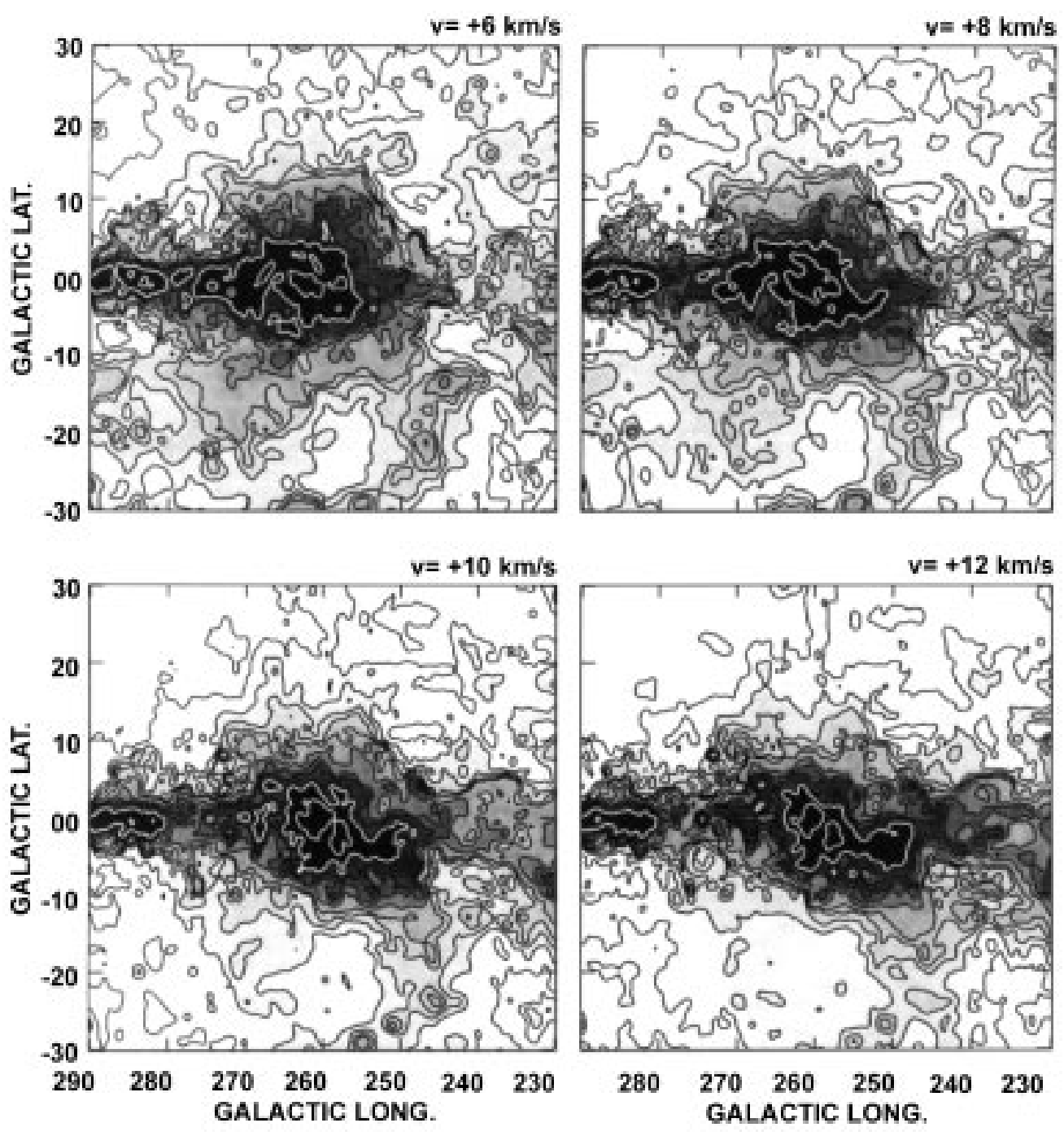

Fig. 2. continued

Vela Shell seems to be the infrared counterpart of the thick HI shell. The IR emission is probably due to the presence of shock heated gas. The present results are consistent with Braun's (1985) findings in the SNR IC443. No HI counterpart is found for the IRAS Vela Shell as a whole.

The Gum nebula has also been studied in X-rays by Leahy et al. (1992), using the HEAO-1 data in the range 0.1 to $3 \mathrm{keV}$. In this study, the authors adjusted for the $\mathrm{X}$-ray emission a single temperature model for a hot optically thick gas. The results were consistent with a uniform $\mathrm{X}$-ray emitting plasma with $T \sim 610^{5} \mathrm{~K}$. The estimated temperature agrees with that expected from the interior of a SNR, according to Chevalier's model (1974). The observed X-ray source region is not completely coincident with the extent of the Gum nebula in $\mathrm{H} \alpha$. Thus, Leahy et al. (1992) suggest that the origin of the X-ray emission may be ascribed to a SNR located within the Gum nebula but not filling it entirely.

\subsection{Origin of the Gum nebula}

\subsubsection{The thick HI shell}

Based on HI data, Dubner et al. (1992) have investigated the thick HI shell in connection with the Gum nebula. They have found that the strong stellar winds of $\zeta$ Puppis, an O4If-type star, and $\gamma^{2}$ Velorum, a Wolf-Rayet spectroscopic binary (WC8+O9I), may have provided enough energy to the ISM to equal the kinetic energy acquired by the thick HI shell. However, by applying the model of Chevalier (1974), they also show that the thick HI shell is consistent with a model of an old SN explosion occurred $2.610^{6}$ years ago. They suggest that this explosion could have originated the Gum nebula. Observations at other wavelengths also support the hypothesis of a SNR. In conclusion, Dubner et al. (1992) propose that the gas distribution was originally perturbed by a SN explosion and the 

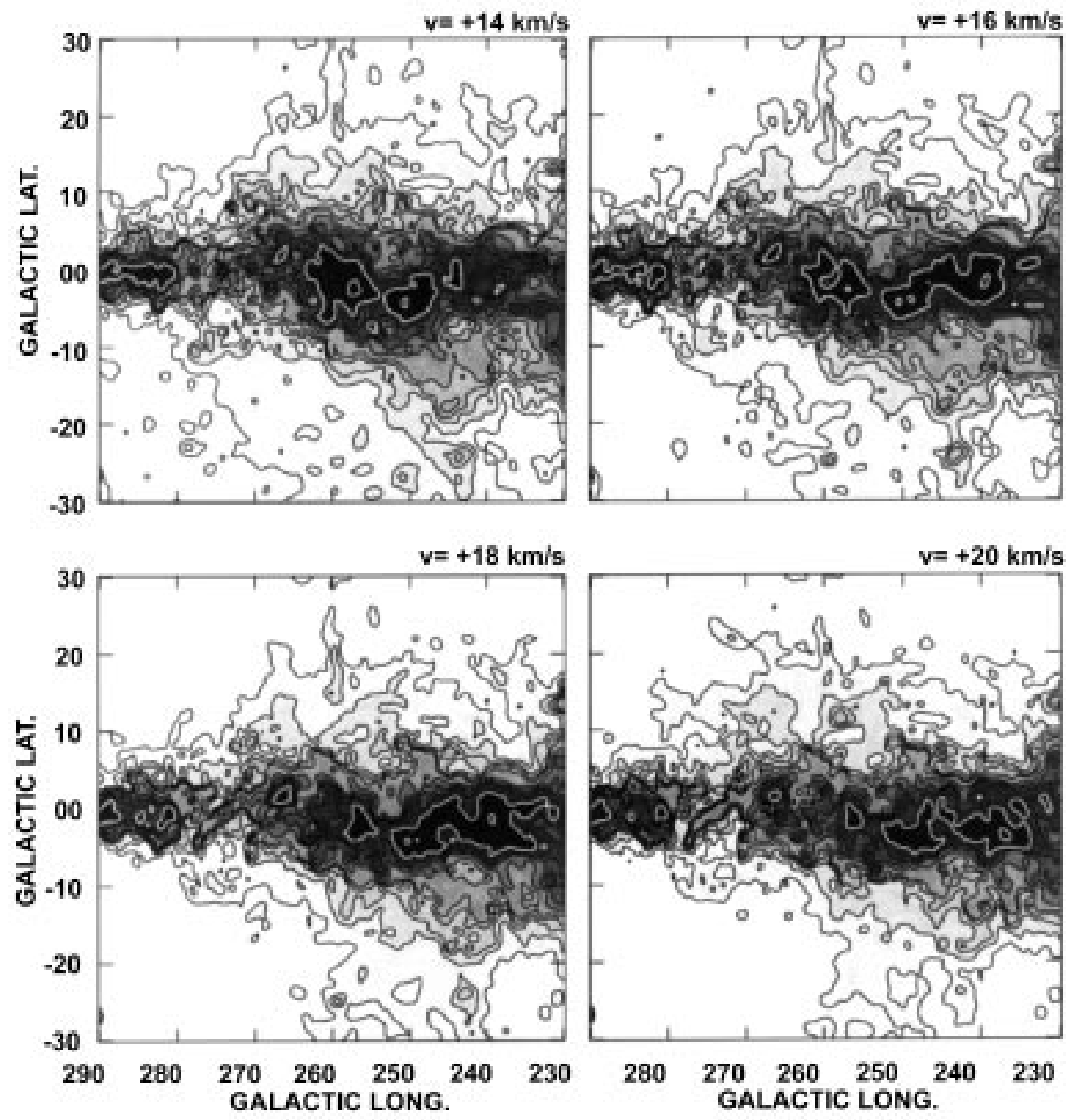

Fig. 2. continued

hollowed shape is now maintained by the stellar winds of the hot massive stars $\zeta$ Puppis and $\gamma^{2}$ Velorum.

\subsubsection{Stellar winds}

In order to find out the origin of the giant HI disk shown in this paper, we first investigate the hypothesis of a winddriven bubble blown by the massive stars present in the region. For this purpose, we have applied the model of Weaver et al. (1977) using typical parameters for O and WR stars, since it is well known that these type of stars have very powerful stellar winds capable of creating large cavities surrounded by HI shells (Cappa de Nicolau \& Niemela 1984; Cappa de Nicolau et al. 1988; Dubner et al. 1990; Niemela \& Cappa de Nicolau 1991).

To estimate the original ambient density, it was assumed that the present swept-up mass was uniformely distributed into a spherical volume of $150 \mathrm{pc}$ in radius. Thus, an initial density of $\sim 0.5 \mathrm{~cm}^{-3}$ was derived. Combining this parameter with the typical mass loss rate and terminal velocity of $\mathrm{O}$ stars, it follows that a bubble could have reached that extent after $910^{6}$ years. This value is almost twice the typical lifetime of $510^{6}$ yrs of an O star. If we assume that the star blowing the bubble was a WR star, the same estimate yields an age of $\sim 410^{6}$ years old for the bubble, again much older than the lifetime of a WR star, typically $510^{5}$ yrs (Maeder 1991). In this latter case, the numbers can be reconciled if we assume that both the WR star and its O-type precursor are responsible for the creation of the big bubble.

The energy provided by $\mathrm{O}$ and WR stars during their entire lives is of the order of $510^{50} \mathrm{ergs}$, as can be computed using typical values for their mass loss rates, terminal wind velocities and mean lifetimes (Abbott 1982; Garmany et al. 1981). By applying the energy conserving model for wind driven bubbles (McCray 1983), it can be assumed that up to $20 \%$ of the total energy could have been deposited into the surrounding medium as kinetic 


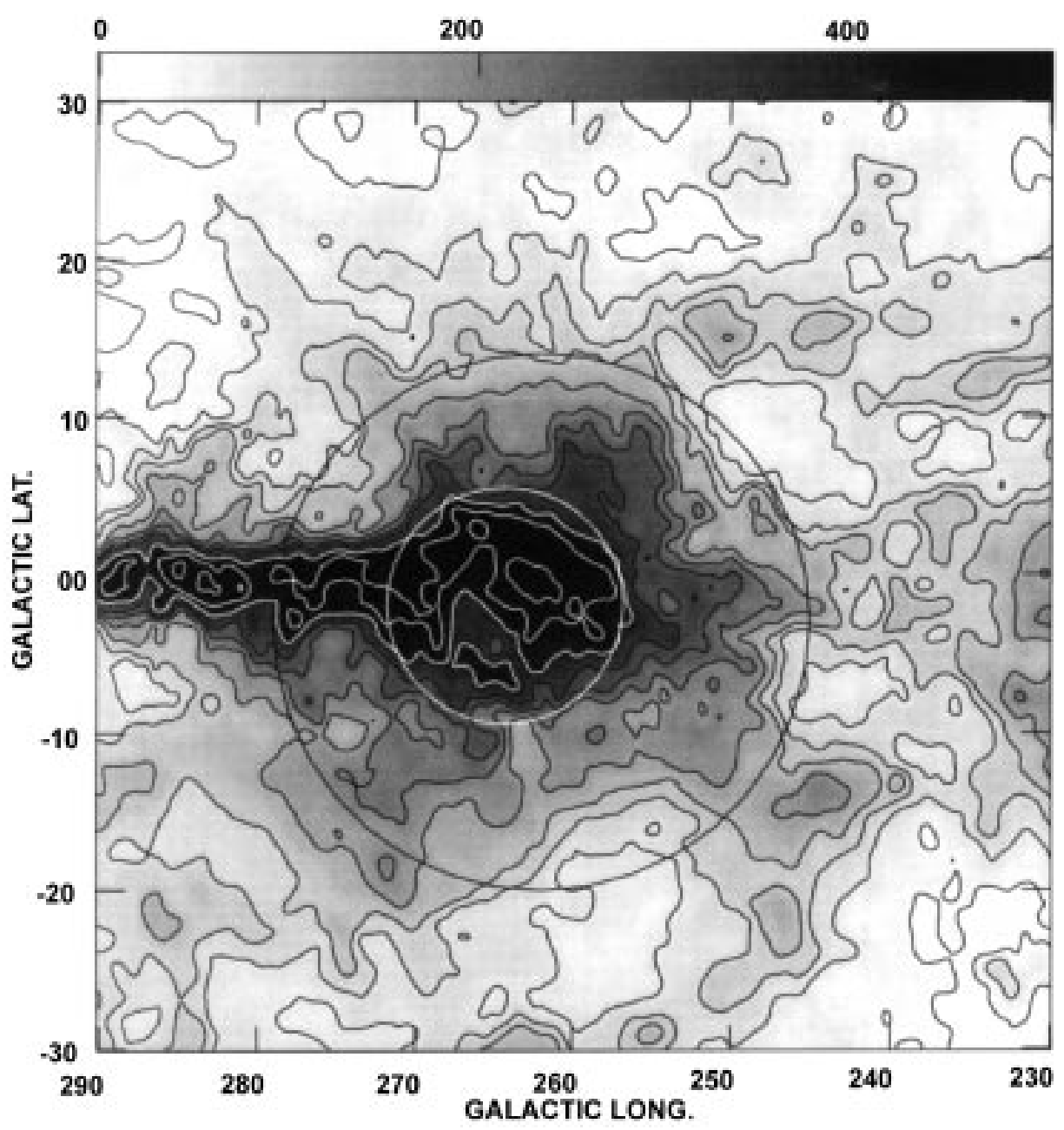

Fig. 3. HI integrated map between 0 and $+10 \mathrm{~km} \mathrm{~s}^{-1}$. Contours are given in brightness temperature, and increase in steps of $25 \mathrm{~K}$ in the interval $25-100 \mathrm{~K}$, and in steps of $50 \mathrm{~K}$ from $100 \mathrm{~K}$ to $300 \mathrm{~K}$. White contours at 400,500 and $600 \mathrm{~K}$ are included. The greyscale is indicated in the wedge on top of the image. The small (white) and the large (black) circles show the approximate locations of the thick HI shell and the giant HI disk, respectively

energy. In both cases, the estimated kinetic energy originating in the star is higher than the kinetic energy associated with the giant $\mathrm{HI}$ disk.

As for the candidate massive early-type stars, together with the two well known massive stars $\zeta$ Pup and $\gamma^{2}$ Vel, which lie near the center of the Gum nebula and at the same distance, there is another O-type star: $\mathrm{CD}-474551$, at a distance of $460 \mathrm{pc}$, placed at $l=267.58^{\circ}, b=-1.22^{\circ}$ (Cruz-González et al. 1974). Based on the above calculation, we conclude that the combined action of these stars can plausibly explain the origin of the giant HI disk. As an alternative explanation, we will also analyze the disk as being created by repeated supernova explosions from an $\mathrm{OB}$ association.

\subsubsection{A "supershell"?}

Multiple explosions can act in concert to compress the ambient interstellar gas into giant expanding shells called "supershells". Supershells have typical radii of $R_{\mathrm{S}} \geq 100 \mathrm{pc}$ and ages of the order of $10^{7}$ years. McCray (1988) estimates that if a typical OB cluster has initially $N_{*} \sim 20$ stars with $M_{*}>7 M_{\odot}$ (i.e., massive stars capable of exploding), then, after explosions have started, a supershell will begin to be created with a radius evolving as

$$
R_{\mathrm{S}}=97 \mathrm{pc}\left[N_{*} E_{51} / n_{0}\right]^{1 / 5} t_{7}^{3 / 5},
$$

where $E_{51}$ is the energy of a $\mathrm{SN}$ explosion in units of $10^{51} \mathrm{ergs}, n_{0}$ is the initial ambient density and $t_{7}$ is the age of the supershell in units of $10^{7}$ years. Application of McCray's model to the giant HI disk produces an age of $\sim 610^{6} \mathrm{yrs}$. This model would be compatible with an expansion velocity of about $14 \mathrm{~km} \mathrm{~s}^{-1}$.

According to this model, our giant HI disk is far from becoming gravitationally unstable, since it should be at least $\sim 310^{7}$ years old. On the contrary, it is possible 


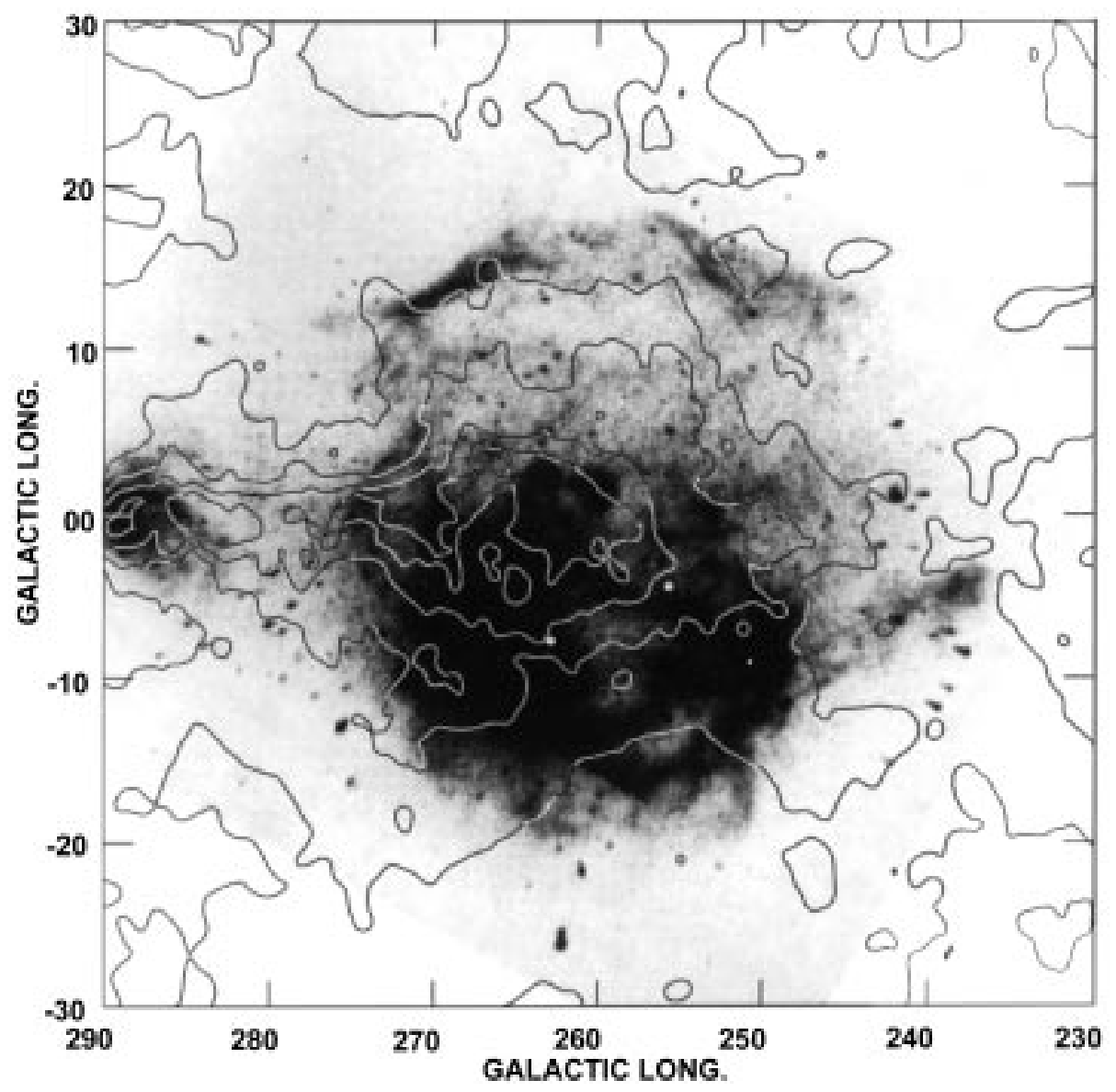

Fig. 4. H $\alpha$ optical image (Sivan 1974) overlapped with the HI contours at 30, 100, 200, 350 and 500 K. The white stars indicate the positions of the stars $\zeta$ Puppis and $\gamma^{2}$ Velorum

that hydrodynamical Rayleigh-Taylor instabilities have occurred. Tenorio-Tagle et al. (1990a) have found that in the presence of a hot halo, the upper part of the remnants will "blowout" because of Rayleigh-Taylor instabilities, and the blowout will result in the formation of chimneys from which ejecta will stream into the halo. As a consequence, the shell as a whole would decelerate due to the loss of internal pressure. The age at which the blowout is produced, about $510^{6}$ years (Tenorio-Tagle et al. 1990b), is consistent with the age we have estimated for the supershell. A blowout would also explain the fact that the observed expansion velocity is lower than the $14 \mathrm{~km} \mathrm{~s}^{-1}$ predicted by McCray's (1988) model.

In the maps shown in Fig. 2, a feature quite similar to a chimney at $l \simeq 260^{\circ}, b>15^{\circ}$ can be seen between +10 and $+20 \mathrm{~km} \mathrm{~s}^{-1}$. If this feature is actually related to the giant HI disk, there is an inconsistency with the kinematic velocity, since it appears at velocities higher than the systemic velocity. However, this apparent incompatibility can be explained with the following argument: the gas streaming upwards through an aperture in the shell to the halo will have a velocity $V$ substantially higher than the expansion velocity of the supershell. If the apperture is located at a latitude $b$ from the galactic plane, the observer will only detect the radial component $v_{\mathrm{r}}$ of the escape velocity $V$, given by $v_{\mathrm{r}}=V \sin b$. Since the feature is most clearly seen between $v=+12$ and $+18 \mathrm{~km} \mathrm{~s}^{-1}$, an LSR velocity $v=+15 \pm 3 \mathrm{~km} \mathrm{~s}^{-1}$ can be adopted, from which $v_{\mathrm{r}}=10 \pm 3 \mathrm{~km} \mathrm{~s}^{-1}$, assuming that the systemic velocity is exactly known. The location of the apperture is $b \simeq 15^{\circ} \pm 2^{\circ}$. Thus, the escape velocity can be estimated to be $V=40 \pm 20 \mathrm{~km} \mathrm{~s}^{-1}$, consistent with the predictions of Tenorio-Tagle et al. (1990a).

The two upper wings in the $\mathrm{H} \alpha$ image of the Gum nebula converge to the feature possibly associated with a chimney. This coincidence supports the hypothesis that the giant $\mathrm{HI}$ disk can be explained as a supershell, and reinforces the contention of the existence of a physical association of the giant HI disk with the Gum nebula. The $\mathrm{H} \alpha$ emission probably arises from the ionization of the hydrogen contained in the shell, where the ionization flux would have been provided by $\zeta$ Pup, $\gamma^{2}$ Vel and the O star CD-474551. Concerning the origin of the explosions which created the supershell, they can be atributed to the 
stars belonging to the relatively aged $\mathrm{OB}$ association Vela OB2, located at $\sim 450 \mathrm{pc}$, which is on the verge of disintegration (Sahu 1992).

A signature of the multiple SN explosions occurred in the past within the Gum nebula can be given by the presence of five pulsars with distances between 0.44 and $0.66 \mathrm{kpc}$ (Manchester \& Taylor 1981), although two of them are older than the supershell, according to the age obtained using the model of McCray (1988).

In summary, we propose that the Gum nebula may be a supershell created by the multiple explosions undergone by stars belonging to the Vela OB2 association. The thick $\mathrm{HI}$ shell fits in this context as the remnant of a late explosion which also contributed to the creation of the Gum nebula. The Vela SN explosion cannot have entered into the formation of the Gum nebula because of its young age, but on the contrary, its evolution may have been influenced by inhomogenities created in the interstellar medium by the past explosions, like the thick HI shell.

\subsection{The southern shell}

It has already been mentioned in Sect. 3 that the southern part of the giant HI disk has been distorted by the presence of a $\sim 28^{\circ}$ diameter shell, centered at $l \simeq 257^{\circ}, b \simeq-23^{\circ}$. The expansive effects of this shell can be seen between -10 and $+14 \mathrm{~km} \mathrm{~s}^{-1}$, and the maximum size is reached at $v \simeq+4 \mathrm{~km} \mathrm{~s}^{-1}$. In Fig. 5, a map of this structure integrated between $v=-10$ and $+14 \mathrm{~km} \mathrm{~s}^{-1}$ is shown. At $l=257^{\circ}, b=-23^{\circ}$, the galactic rotation model of Fich et al. (1989) predicts a distance of $240 \mathrm{pc}$ for a systemic velocity of $+4 \mathrm{~km} \mathrm{~s}^{-1}$. However, it should be borne in mind that a $1 \mathrm{~km} \mathrm{~s}^{-1}$ difference in the systemic velocity produces a change of nearly $150 \mathrm{pc}$ in distance at this longitude. Thus, since the usage of galactic rotation models may not yield a precise result, we will try to get an independent estimate for the distance, based on the search of an agent capable of having evacuated the cavity. The only powerful nearby star located in the inner region of the HI hole is the O5/7-type star HD 49798, lying at a distance of 570 pc (Cruz-González et al. 1974). This star is indicated in Fig. 5 as a star. We will suppose that the shell has been created by the star HD 49798 and therefore lies also at a distance of $570 \mathrm{pc}$. Under this assumption, the radius of the shell can be estimated to be $\sim 142 \mathrm{pc}$. The reliability of our hypothesis will be tested below using the model of Weaver et al. (1977).

The shell is open towards the lower density region, opposite to the galactic disk, while the expansion has obviously been slowed down in the direction of the denser region. The eccentric position of the star agrees with such an anisotropic expansion, though optical measurements should reveal if the eccentricity is rather due to a proper motion of the star. The southern part of the shell extends beyond the area surveyed in this paper, thus the mass es- timates will be based on the available data and considered as a lower limit.

The amount of matter present in the shell is $M \geq 1.510^{5} d^{2} M_{\odot}$. Assuming that $d \simeq 0.57 \mathrm{kpc}$, then $M \geq 510^{4} M_{\odot}$. Adopting an expansion velocity of $10 \pm 2 \mathrm{~km} \mathrm{~s}^{-1}$, the expansion kinetic energy is above $510^{49}$ ergs. According to the calculations carried out in the previous section, an $\mathrm{O}$ star could have provided this energy to the interstellar medium through stellar winds. If it is assumed that the mass contained in the shell was initially uniformely distributed throughout a sphere of $142 \mathrm{pc}$ radius, the initial ambient density should have been higher than $0.16 \mathrm{~cm}^{-3}$. Application of the model of Weaver et al. (1977) implies that the O star HD 49798 should have spent more than $5.610^{6}$ yrs blowing the bubble. This time is compatible with a typical O-star lifetime.

There has not been found any correlation between this $\mathrm{HI}$ structure and the $\mathrm{H} \alpha$ emission. In particular, no optical filaments appear towards the most dense regions, as would be expected if there were important energy dissipation due to the interaction between the HI shell and the Gum nebula.

Based on the above results, it can be concluded that the southern HI shell was probably created by the stellar winds of the O-type star HD 49798, and that its age is close to the age of the Gum nebula. The interaction between these two structures can be noticed through the distortion of the isolines at the region of overlap.

\section{Conclusions}

In this paper, we have presented neutral hydrogen $21 \mathrm{~cm}$ images of a $60^{\circ} \times 60^{\circ}$ region covering completely the area subtended by the optical image of the Gum nebula. The maps have been constructed combining data extracted from the surveys performed by Weaver \& Williams (1973), Heiles \& Habing (1974), Colomb et al. $(1977,1980)$ and Strong et al. (1982). The main result was the discovery of a giant HI disk which holds the optical nebula in its interior. This is the first study in which a structure with the same dimensions of the optical image of the Gum nebula is detected in another spectral region. Another case of a huge HI structure detected in connection with an optical supershell in our galaxy is the Cyg OB1/OB3 supershell (Dewdney \& Lozinskaya 1994).

We cannot unequivocally conclude that the HI disk is associated with the optical Gum nebula. However, we believe that there is a reasonable probability that both structures arise from the same physical mechanism. We investigated the hypothesis of the Gum nebula being a supershell driven by multiple supernova explosions, in the context of the theory of McCray (1988). The distance to the Gum nebula was estimated to be $\sim 500 \mathrm{pc}$, thus having a radius of $\sim 150 \mathrm{pc}$. Based on a supershell model, an age of about $610^{6}$ yrs was derived. A possible signature of a "blowout" towards the northern extreme of the shell was 


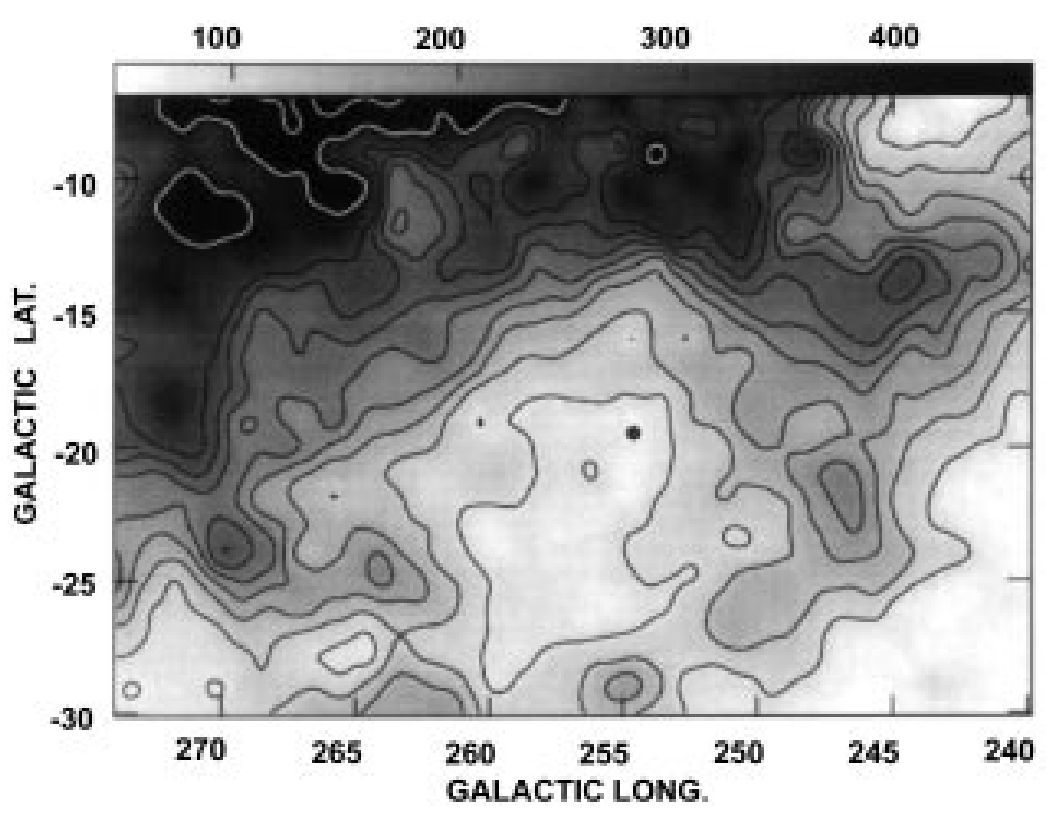

Fig. 5. Integrated map between -10 and $+14 \mathrm{~km} \mathrm{~s}^{-1}$ of the shell lying to the south of the Gum nebula. Contours are at 90 , $120,150,180,210,240270,300,400$ and $500 \mathrm{~K}$. The greyscale is shown in the edge on top of the image. The star indicates the position of the O-type star HD 49798

detected at $v \simeq+15 \mathrm{~km} \mathrm{~s}^{-1}$. This feature, which has the appearence of a chimney, is consistent with the locus of convergence of the two upper $\mathrm{H} \alpha$ wings and agrees with the theoretical predictions of Tenorio-Tagle et al. (1990a).

A number of pulsars enclosed by the supershell may be indicative of the occurrence of multiple ancient explosions in the region. Additional support for the hypothesis of repeated supernova explosions in the past is given by the thick HI shell reported by Dubner et al. (1992), which was proposed to be an old SNR based on different wavelengths results. The source of the explosions can be attributed to the Vela OB2 association. We suggest that the $\mathrm{H} \alpha$ emission arises from the ionization of the HI disk produced mainly by the early stars $\zeta$ Pup, $\gamma^{2}$ Vel and CD-474551, located inside the supershell.

The Gum nebula appears to be an old, now decelerated supershell. If its low expansion velocity arises from the SN explosions having turned off long ago, then the bubble interior should have cooled and thus the corresponding hole in the HI distribution no longer exists (Vader \& Chaboyer 1995). It must be mentioned, however, that a wind driven bubble model (Weaver et al. 1977) is marginally compatible with observations, both using kinematical and energetic arguments.

At smaller scales, it was found that the $\mathrm{H} \alpha$ and the $\mathrm{HI}$ emission are poorly correlated. In particular, the southern appearence of the Gum nebula in $\mathrm{H} \alpha$ is not reproduced in the $21 \mathrm{~cm}$ image, where the contours appear distorted by another shell. We have shown that this southern shell is consistent with an $\mathrm{HI}$ bubble blown by the O-type star HD 49798. Applying the model of Weaver et al. (1977), an age of $5.610^{6}$ yrs was obtained. It follows, then, that the Gum nebula and this southern shell were born at the same time and may have interacted with one another, as suggested by the deformation observed in the distribution of the neutral gas.

Acknowledgements. We would like to thank J. P. Sivan for providing us his $\mathrm{H} \alpha$ photographs of the Gum nebula.

\section{References}

Abbott D.C., 1982, ApJ 263, 723

Beuermann K.P., 1973, Ap\&SS 20, 27

Brandt J.C., Stecher T.P., Crawford D.L., Maran S.P., 1971, ApJ 163, L99

Braun R., 1985, "The interaction of supernovæ with the interstellar medium", Doctoral Thesis, Leiden University

Cappa de Nicolau C.E., Niemela V.S., 1984, AJ 89, 1398

Cappa de Nicolau C.E., Niemela V.S., Dubner G.M., Arnal E.M., 1988, AJ 96, 1671

Chanot A., Sivan J.P., 1983, A\&A 121, 19

Chevalier R.A., 1974, ApJ 188, 501

Colomb F.R., Pöppel W.G.L., Heiles C., 1977, A\&AS 29, 89

Colomb F.R., Pöppel W.G.L., Heiles C., 1980, A\&AS 40, 47

Cruz-González C., Recillas-Cruz E., Costero R., Peimbert M., Torres-Peimbert S., 1974, Rev. Mex. Astron. Astrofís. 1, 211

Dewdney P.E., Lozinskaya T.A., 1995, AJ 108, 2212

Dubner G.M., Niemela V.S., Purton C.R., 1990, AJ 99, 857

Dubner G.M., Giacani, E.B., Cappa de Nicolau C.E., Reynoso E.M., 1992, A\&AS 96, 505

Fich M., Blitz L., Stark A.A., 1989, ApJ 342, 272

Franco G.A.P., 1990, A\&A 227, 499 
Garmany C.D., Olson G.L., Conti P.S., van Steenburg M., 1981, ApJ 250, 660

Gum C.S., 1956, Observatory 76, 150

Heiles C., Habing H.J., 1974, A\&AS 14, 1

Hippelein H.H., Weinberger R., 1975, A\&A 43, 405

Leahy D.A., Nousek J., Garmire G., 1992, ApJ 385, 561

Maeder A., 1991, IAU Symp. 143, "WR stars and interrelations with other massive stars in galaxies". In: van der Hutch K.A. and Hydayat B. (eds.) p. 445

Manchester R.N., Taylor J.H., 1981, AJ 86, 1953

McCray R., 1983, in "Highlights of Astronomy", Vol. 6, West R.M. (ed.) p. 565

McCray R., 1988, in "Supernova Remnants and the Interstellar Medium" (IAU Coll. 101), Roger R.S. and Landecker T.L. (eds.). Cambridge University Press, Cambridge, p. 447

McGee R.X., Murray J.D., Milton J.A., 1963, Aust. J. Phys. 16,136

McGee R.X., Milton J.A., Wolfe W., 1966, Aust. J. Phys. Astrophys. Suppl. 1, 3
Niemela V.S., Cappa de Nicolau C.E., 1991, AJ 101, 572

Reynolds S.P., 1976a, ApJ 203, 151

Reynolds S.P., 1976b, ApJ 206, 679

Sahu M.S., 1992, "A study of the ISM in Puppis-Vela including the Gum nebula", Doctoral Thesis, Groningen University

Sivan J.P., 1974, A\&AS 16, 163

Srinivasan M., Pottasch S.R., Sahu K.C., Pecker J.C., 1987, Messenger 50, 11

Strong A.W., Riley P.A., Osborne J.L., Murray J.D., 1982, MNRAS 201, 495

Tenorio-Tagle G., Bodenheimer P., Franco J., Różyczka M., 1990a, MNRAS 244, 563

Tenorio-Tagle G., Różyczka M., Bodenheimer P., 1990b, A\&A 237, 207

Vader J.P., Chaboyer B., 1995, ApJ 445, 691

Wallerstein G., Silk J., Jenkins G.B., 1980, ApJ 240, 834

Weaver H., Williams D.R.W., 1973, A\&AS 8, 1

Weaver R., McCray R., Castor J., Shapiro P., Moore R., 1977, ApJ 218, 377 